\title{
Les fausses nouvelles et les attaques des négateurs scientifiques contre les vaccins. Que pouvez-vous faire?
}

\author{
Noni E MacDonald ${ }^{1 \star}$
}

\section{Résumé}

La mésinformation et la désinformation (« fausses nouvelles ») sur les vaccins sont contagieuses : elles se déplacent plus vite et plus loin que la vérité. Les conséquences sont graves, menant à des effets négatifs sur les décisions en matière de santé, y compris l'acceptation des vaccins, et sur la confiance dans les conseils d'immunisation fournis par les professionnels de la santé publique ou des soins de santé. Le présent article donne un aperçu des stratégies fondées sur des données probantes pour traiter les négateurs du vaccin en public, en pratique clinique et dans des situations sociales. De plus, une stratégie visant à aider à différencier les négateurs du vaccin et les personnes qui refusent simplement un vaccin dans une clinique ou une pratique est fournie. Cinq tactiques sont largement utilisées par les négateurs des vaccins : complots; faux experts; sélectivité; attentes impossibles; et fausse représentation et fausse logique. La reconnaissance et la compréhension de ces tactiques peuvent contribuer à protéger contre la désinformation et la propagande négationniste scientifique. Mettre en évidence le consensus solide des sciences médicales sur l'innocuité et l'efficacité des vaccins est également utile. Il est crucial de choisir avec soin et sagesse ce qu'il faut dire, et vous exprimer, que vous soyez à un dîner, avec des amis ou dans votre clinique ou cabinet médical. Ne pas vous exprimer suggère que vous êtes d'accord avec la mésinformation. Il est de plus en plus important que les fournisseurs de soins de santé reconnaissent et traitent la mésinformation en utilisant des stratégies fondées sur des données probantes, car l'arrivée des vaccins contre la maladie à coronavirus de 2019 (COVID-19) devrait intensifier encore la rhétorique de mésinformation et de désinformation sur les vaccins. Les fournisseurs de soins de santé doivent se préparer et agir dès maintenant pour lutter contre le tsunami de la mésinformation vaccinale.
Cette oeuvre est mise à la disposition selon les termes de la licence internationale Creative Commons Attribution 4.0

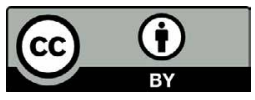

Affiliation

${ }^{1}$ Département de pédiatrie, Dalhousie University, IWK Health Centre, Halifax, NS

\section{*Correspondance : \\ noni.macdonald@dal.ca}

Citation proposée : MacDonald NE. Les fausses nouvelles et les attaques des négateurs scientifiques contre les vaccins. Que pouvez-vous faire? Relevé des maladies transmissibles au Canada 2020;46(11/12):486-90.

https://doi.org/10.14745/ccdr.v46i1112a11f

Mots-clés : négateur de la science, vaccin, mésinformation, désinformation

\section{Introduction}

Jamais auparavant le public n'a été aussi bombardé par l'information, et il n'a jamais été aussi difficile de savoir quoi et qui croire. L'importance essentielle de ce problème est bien illustrée par l'Organisation mondiale de la santé (OMS) qui éclaire brillamment l'infodémie pandémique sur la maladie à coronavirus en 2019 (COVID-19). Une infodémie désigne une diffusion rapide et étendue de renseignements exacts et inexacts. La mésinformation (information fausse, mais non créée dans l'intention de causer du tort) et la désinformation (ou "fausse information "; les informations fausses et délibérément créées pour causer du tort) se déplacent plus rapidement et plus loin que la vérité $(2,3)$. Les négateurs scientifiques, y compris les négateurs de la science des vaccins, disposent maintenant d'une plateforme solide et très efficace, le Web, à partir de laquelle ils peuvent présenter leurs produits en faillite scientifique (4). Nous, qui comprenons la rigueur de la science et connaissons les preuves à l'appui de l'immunisation pour la santé et le bien-être, sommes souvent révoltés par les mensonges promulgués et, trop souvent, acceptés et suivis par les citoyens. Par exemple, aux États-Unis, la variation de l'absorption du vaccin contre le papillomavirus humain (VPH) à travers le pays s'explique mieux par l'exposition à des tweets sur le VPH que par les données de la classe socio-économique (5). 


\section{Pourquoi cela se produit-il?}

Malheureusement, nous prenons tous la plupart de nos décisions en fonction de nos croyances et non en fonction de preuves scientifiques soigneusement pesées (6). Nous voyons et entendons ce que nous croyons, plutôt que de croire ce que nous voyons et entendons (7). Nous sommes fortement influencés par ce que nous pensons que les autres autour de nous (nos réseaux sociaux) font ou attendent de nous. Nous voyons la causalité dans les coïncidences et nous préférons l'anecdote et les histoires aux données et aux preuves scientifiques.

L'objectif de cet article est d'attirer l'attention sur l'importance des fausses nouvelles et des attaques de négateurs scientifiques contre les vaccins à l'ère des médias sociaux. Il décrira les tactiques utilisées par les négateurs scientifiques et mettra en évidence les stratégies que les prestataires de soins de santé peuvent utiliser dans leur bureau ou leur clinique lorsqu'ils rencontrent une personne refusant un vaccin ou un négateur scientifique, ainsi que l'adresse URL d'un site Web de I'OMS pour un rapport sur la mésinformation trouvée en ligne.

Il s'agit du neuvième article produit par le Centre canadien de ressources et d'échange de données probantes sur la vaccination (CANVax) dans la série de résumés de CANVax. Ce centre comprend un groupe de professionnels multidisciplinaires qui identifient et créent des ressources utiles pour favoriser l'adoption des vaccins (8).

\section{Que pouvez-vous faire?}

Que pouvez-vous faire face à ce tsunami de mésinformation et de désinformation qui façonne les croyances négatives sur l'immunisation chez le grand public, les patients et même, parfois, chez nos collègues professionnels? La mésinformation est en effet le problème de tout le monde maintenant (9). Les conséquences sont graves, menant à des effets négatifs sur les décisions en matière de santé, y compris l'acceptation des vaccins, et sur la confiance dans les conseils d'immunisation fournis par les professionnels de la santé publique ou des soins de santé. Cette incidence de la mésinformation et de la désinformation deviendra encore plus importante lorsque les vaccins contre la COVID-19 arriveront, avec une augmentation prévue de la mésinformation vaccinale (10). Si des contremesures ne sont pas prises, le mouvement anti-vaccin pourrait submerger les voix pro-vaccin en ligne (10). Vous pouvez et devez aider à combattre ce tsunami de mésinformation vaccinale.

\section{Préparez-vous : connaître et reconnaître les tactiques utilisées par les négateurs des vaccins}

Connaître les cinq tactiques largement utilisées, souvent avec une grande vigueur, par les négateurs des vaccins vocaux sur le Web, dans les médias traditionnels et dans les apparitions publiques (11):

- Les complots - les compagnies pharmaceutiques, le gouvernement, le système de santé - choisissez votre bouc émissaire - sont là pour tromper le grand public; ils cachent l'information, mentent et dissimulent "la vérité "

- Les faux experts - citer ou utiliser de faux experts et dénigrer vigoureusement, voire décrier, de vrais experts

- Sélectivité - renvoie à des documents obscurs ou discrédités qui étayent leur argument, mais omettent la vaste science qui la réfute

- Attentes impossibles - le vaccin doit être sûr et efficace à $100 \%$ - et pourtant aucune intervention médicale n'est à 100 \% sûre et efficace

- Fausse représentation et fausse logique - sauter à une conclusion erronée et utiliser des analogies fausses ou illogiques

Il est intéressant de noter qu'une fois que vous connaissez ces tactiques, elles sont faciles à reconnaître, comme en témoignent les plaintes pour de fausses nouvelles et la désinformation qui apparaissent presque quotidiennement dans les médias traditionnels et sociaux.

\section{Apprenez à vos patients à reconnaître les tactiques des négateurs scientifiques}

Il y a des études scientifiques qui ont montré qu'une façon de protéger le public contre les fausses nouvelles et les négateurs scientifiques est d'enseigner au public les tactiques utilisées, et pas seulement de corriger la mésinformation scientifique présentée (12). Si un site Internet est la source de mésinformation, pensez à le signaler sur le site Web de l'OMS "Comment signaler une mésinformation en ligne » (13).

\section{Souligner le consensus scientifique}

Souligner qu'il existe un consensus scientifique sur les avantages et la valeur de l'immunisation est également utile (14) lorsqu'il s'agit de réagir aux fausses nouvelles sur les vaccins et l'immunisation. Partagez vos sources d'information exacte et de qualité sur les vaccins avec vos patients. Ces étapes ne convaincront pas le négateur vocal sur le vaccin, mais elles sont utiles à ceux qui hésitent dans votre public cible, vos patients et le grand public. 


\section{Lutter contre les négateurs vocaux des vaccins en public}

Le Bureau régional pour l'Europe de l'OMS a élaboré des directives efficaces sur la façon de traiter publiquement les négateurs vocaux des vaccins $(15,16)$. Il ne s'agit pas d'une tâche facile, mais il s'agit d'une tâche importante à entreprendre si le négateur vocal de la science du vaccin a ou pourrait avoir une incidence négative importante sur la confiance dans l'immunisation dans votre collectivité.

L'orientation de l'OMS s'adresse principalement aux porteparole des autorités sanitaires qui veulent se préparer à une manifestation publique avec un négateur vocal des vaccins, et donne des conseils sur qui devrait être le porte-parole, quoi faire et ne pas faire dans les communications verbales et non verbales, comment se comporter dans une discussion passionnée et comment se protéger. Elle fournit des stratégies utiles et étayées par des données probantes si vous devez vous faire demander de parler en public.

Un point important : ne participez pas à une discussion publique si vous n'êtes pas formé aux médias.

\section{Stratégies visant à discuter avec un négateur de la vaccination en pratique clinique}

\section{Différenciation entre un négateur scientifique et un simple refus}

La première et très importante étape consiste à déterminer si le patient qui ne veut pas prendre le vaccin est un négateur scientifique ou un simple réfractaire. Vous pouvez rapidement essayer de résoudre ce problème en demandant : "Qu'est-ce qu'il faudrait pour vous amener à un "oui" pour accepter ce vaccin? » Le simple réfractaire peut s'arrêter, penser et nommer la préoccupation. Cela est encore plus probable si vous avez un bon rapport et une relation de confiance avec le patient. En revanche, vous obtiendrez une réaction très différente du négateur de la science du vaccin. Ils commencent le plus souvent par une longue liste de préoccupations et veulent travailler dur pour vous convaincre de leur point de vue. Soyez méfiant.

\section{Stratégies à utiliser pour traiter les refus de vaccin}

Les personnes refusant des vaccins ont habituellement une ou deux préoccupations principales. Pour répondre à cette préoccupation, tenez compte des conseils suivants :
- Ne faites pas de la séance une « décharge des connaissances ", car il est rarement utile d'accabler la personne qui refuse avec l'information et peut en fait finir par soulever des préoccupations à propos desquelles elle n'était pas auparavant inquiète

- Ne passez pas de temps à réfuter les mythes, car cela ne change pas les attitudes à l'égard de l'immunisation (17); de plus, il se peut que ce soit le mythe dont la personne se souvient plutôt que des renseignements exacts

- La mini-entrevue motivationnelle est une stratégie plus utile pour mieux comprendre les préoccupations et faire progresser le patient vers l'acceptation $(18,19)$; I'OMS a un court module de formation sur cette technique de vaccination que vous pourriez trouver utile (20)

\section{Stratégies à utiliser pour lutter contre les négateurs scientifiques}

Le terme "négateur des vaccins » désigne un membre d'un sous-groupe à l'extrémité du continuum d'hésitation; celui qui a une attitude très négative à l'égard de la vaccination et qui n'est pas ouvert à un changement d'avis quoi que disent les données scientifiques (11). Il y a plusieurs points à retenir lorsqu'il s'agit de discuter avec les négateurs scientifiques.

- N'entamez pas un débat avec le négateur; c'est un piège qui gaspille du temps

- Déclarez que la science est clairement derrière la vaccination. Encore une fois, faites-le sans entrer dans un débat : il est très peu probable que vous convainquiez le négateur de vos arguments et que vous finissiez probablement par un cycle inutile de " oui, mais »

- Vous pouvez essayer une mini-entrevue motivationnelle comme indiqué ci-dessus. Avec de solides négateurs de la science des vaccins, cela est moins susceptible d'aider par rapport à de simples réfractaires, mais cela vaut la peine d'être essayé

\section{Laisser la porte ouverte}

Que le patient soit un négateur ou une personne qui refuse, s'il choisit de ne pas vacciner son enfant ou lui-même ce jour-là, laissez la porte ouverte à de futures visites et discussions. $\mathrm{Ne}$ les rejetez pas de votre pratique, même si c'est tentant, car cela n'est pas dans l'intérêt du patient ou de la communauté (21). De plus, il est cliniquement important de passer en revue les risques et les responsabilités si le patient choisit de ne pas accepter le ou les vaccins. Le site Web de la Société canadienne de pédiatrie s'occupant des enfants donne des conseils à ce sujet que vous pouvez ensuite personnaliser ou adapter à la situation de votre patient (22).

\section{Ne pas rester silencieux}

Enfin, rappelez-vous de ne pas vous taire lorsque vous êtes confronté à un négateur de la science des vaccins, car votre silence peut être interprété par les autres autour de vous que vous êtes d'accord avec la mésinformation. Choisissez 
soigneusement et sagement quoi dire et exprimez-vous, que ce soit à un collègue de travail ou à un patient ou à un ami. Le public cible n'est pas le négateur, mais les autres autour de vous. N'oubliez pas d'éduquer les autres sur les techniques de mésinformation utilisées et d'aider à vacciner contre les fausses nouvelles et le déni scientifique.

\section{Conclusion}

À la lumière des fausses nouvelles sur les vaccins et les attaques des négateurs scientifiques contre les vaccins qui prolifèrent sur les médias tant traditionnels que sociaux (10), il est essentiel d'apprendre à différencier les véritables négateurs scientifiques des personnes refusant les vaccins, et à identifier les simples réfractaires, qui se sont montrés incertains dans leurs croyances d'acceptation des vaccins par les machinations des négateurs scientifiques. Connaître et utiliser des stratégies appropriées pour les deux groupes donne aux fournisseurs de soins de santé les moyens de traiter adéquatement les situations dans les milieux professionnels et personnels.

\section{Déclaration de l'auteur}

N. E. M. - Conceptualisation, rédaction de l'ébauche originale, révision et édition

\section{Intérêts concurrents}

N. E. MacDonald a reçu des subventions de l'Agence de la santé publique du Canada, de l'Organisation mondiale de la santé, de la Fondation de la recherche en santé de la Nouvelle-Écosse, des Instituts de recherche en santé du Canada, du Réseau canadien de recherche sur l'immunisation et du Conseil de recherches en sciences humaines du Canada. Elle est membre de l'équipe du Centre canadien de recherche et d'échange de données probantes sur la vaccination (CANVax).

\section{Remerciements}

La production des résumés du Centre canadien de ressources et d'échange de données probantes sur la vaccination (CANVax) a été rendue possible grâce à un financement de l'Agence de santé publique du Canada. Merci aux nombreux auteurs, partenaires en immunisation et examinateurs qui contribuent à CANVax.

\section{Financement}

L'élaboration des résumés du Centre canadien de ressources et d'échange de données probantes sur la vaccination est appuyée par le Fonds de partenariat d'immunisation de l'Agence de santé publique du Canada.

\section{Références}

1. World Health Organization. Infodemic management Infodemiology. WHO; 2020 (accédé 2020-10-08). https://www.who.int/teams/risk-communication/ infodemic-management

2. Bauch CT, Galvani AP. Epidemiology. Social factors in epidemiology. Science 2013;342(6154):47-9. DOI PubMed

3. Vosoughi S, Roy D, Aral S. The spread of true and false news online. Science 2018;359(6380):1146-51. DOI PubMed

4. Kata A. Anti-vaccine activists, Web 2.0, and the postmodern paradigm--an overview of tactics and tropes used online by the anti-vaccination movement. Vaccine 2012;30(25):3778-89. DOI PubMed

5. Dunn AG, Surian D, Leask J, Dey A, Mandl KD, Coiera E. Mapping information exposure on social media to explain differences in HPV vaccine coverage in the United States. Vaccine 2017;35(23):3033-40. DOI PubMed

6. Kahan DM. Social science. A risky science communication environment for vaccines. Science 2013;342(6154):53-4. DOI PubMed

7. Dubé È, MacDonald NE. Managing the risks of vaccine hesitancy and refusals. Lancet Infect Dis 2016;16(5):518-9. DOl PubMed

8. Association canadienne de Santé publique. Centre canadien de ressources et d'échange sur les données probantes en vaccination. Ottawa (ON) : CANVax.

https://www.canvax.ca/fr

9. Donavan J, Wardle C. Misinformation is Everybody's Problem Now. Social Science Research Council. Items. Insights from the Social Science. SSRC; August 6, 2020 (accédé 2020-10-08). https://items.ssrc.org/covid19-and-the-social-sciences/mediated-crisis/misinformation-i s-everybodys-problem-now/

10. Burki T. The online anti-vaccine movement in the age of COVID-19. Lancet Digit Health 2020;2(10):e504-5. DOl PubMed

11. Schmid P, MacDonald NE, Habersaat K, Butler R. Commentary to: how to respond to vocal vaccine deniers in public. Vaccine 2018;36(2):196-8. DOI PubMed 
12. van der Linden $S$, Maibach $E$, Cook J, Leiserowitz A, Lewandowsky $S$. Inoculating against misinformation. Science 2017;358(6367):1141-2. DOl PubMed

13. Organisation mondiale de la Santé. Comment signaler les informations erronées en ligne. Genève (Suisse) : OMS; 2020 (accédé 2020-10-08). https://www.who.int/fr/ campaigns/connecting-the-world-to-combat-coronavirus/ how-to-report-misinformation-online

14. van der Linden SL, Clarke CE, Maibach EW. Highlighting consensus among medical scientists increases public support for vaccines: evidence from a randomized experiment. BMC Public Health 2015;15:1207. DOI PubMed

15. World Health Organization Regional Office for Europe. Best practice guidance: How to respond to vocal vaccine deniers in public (2017). WHO; 2017 (accédé 2020-10-08). https:// www.euro.who.int/en/health-topics/disease-prevention/ vaccines-and-immunization/publications/2016/ best-practice-guidance-how-to-respond-to-vocal-vac cine-deniers-in-public-2017

16. Schmid $P$, Betsch $C$. Effective strategies for rebutting science denialism in public discussions. Nat Hum Behav 2019;3(9):931-9. DOl PubMed

17. Horne Z, Powell D, Hummel JE, Holyoak KJ. Countering antivaccination attitudes. Proc Natl Acad Sci USA 2015;112(33):10321-4. DOl PubMed
18. Gagneur A, Lemaître T, Gosselin V, Farrands A, Carrier N, Petit G, Valiquette L, De Wals P. A postpartum vaccination promotion intervention using motivational interviewing techniques improves short-term vaccine coverage: PromoVac study. BMC Public Health 2018;18(1):811. DOI PubMed

19. Gagneur A. L'entrevue motivationnelle : un outil particulièrement efficace pour atténuer la réticence à la vaccination. Relevé des maladies transmissibles au Canada 2020;46(4):104-9. DOI

20. World Health Organization. Immunization, Vaccine and Biologicals. Improving vaccination demand and addressing hesitancy. WHO (modifié 2015-10-16; accédé 2020-06-29). http://www.who.int/immunization/programmes_systems/ vaccine_hesitancy/en/

21. MacDonald NE, Harmon S, Dube E, Taylor B, Steenbeek A, Crowcroft N, Graham J. Is physician dismissal of vaccine refusers an acceptable practice in Canada? A 2018 overview. Paediatr Child Health 2019;24(2):92-7. DOI PubMed

22. Société canadienne de pédiatrie. Soins de nos enfants. Les parents qui choisissent de ne pas faire vacciner leur enfant : les risques et les responsabilités. SCP; août 2016 (accédé 2020-10-08). https://www.soinsdenosenfants.cps.ca/ handouts/when-parents-choose-not-to-vaccinate-risks-andresponsibilities 